Rapid Reviews COVID-19

\title{
Review 2: "Impaired local intrinsic immunity to SARS-CoV-2 infection in severe COVID-19"
}

Alejandro Berrio ${ }^{1}$

${ }^{1}$ Postdoctoral Associate, Duke University, Biology

Published on: Apr 09, 2021

License: Creative Commons Attribution 4.0 International License (CC-BY 4.0). 


\section{$\underline{\text { RR:C19 Evidence Scale rating by reviewer: }}$}

- Strong. The main study claims are very well-justified by the data and analytic methods used. There is little room for doubt that the study produced has very similar results and conclusions as compared with the hypothetical ideal study. The study's main claims should be considered conclusive and actionable without reservation.

$* * * * * * * * * * * * * * * * * * * * * * * * * * * * * * * * * * * * * * * *$

\section{Review:}

Ziegler and collaborators use single-cell genomic data from patients under different classes of COVID-19 progression and controls to investigate which cell types in the human nasopharynx are the primary targets of SARS-CoV-2 infection, and how the coronavirus infection influences the cellular organization of the respiratory epithelium. This is a phenomenon that remains incompletely understood and the authors made an exceptional effort to understand it and identify some of the changes in cell organization in human nasopharynx undergoing different infection states or disease outcomes. Overall, I think this research is strong, and the authors make a careful discussion of their experimental design and its caveats. Claims are very wellsupported by the data and methods used. Decision-makers should consider the claims in this study actionable without reservation based on the methods and

data. Moreover, I don't have significant concerns regarding the implementation of the Seurat and other computational tools for computing RNA Velocity and Pseudotime. In particular, I am very impressed by this manuscript, it is well written and the statistical analysis are sound. Finally, the authors unlock insights that will help to identify what cells and what novel candidate genes would help to predict the severity outcome of the disease. Some highlights of this work are: 1) Massive reorganization of the nasopharyngeal tissue or upper respiratory epithelium. 2) Cells from patients with mild/moderate COVID-19 show induction of genes associated with anti-viral and type I interferon responses, while this response is muted in severe cases. 3) Extraordinary diversity and heterogeneity among SARS-CoV-2 RNA+ host cells. 4) SARS-CoV-2 RNA+ host cells also exhibit an enrichment of genes involved in susceptibility (e.g., CTSL, TMPRSS2) or response (e.g., MX1, IFITM3, EIF2AK2) to infection. The authors also report the upregulation of alarmins S100A8/S100A9 (i.e., calprotectin) among severe and moderate/mild cases relative to controls. 
The manuscript confirms previous work of the current understanding of the effect of SARS-CoV-2 infection on interferon genes. Indeed, the evidence that has been provided will improve the understanding of COVID-19 and the degree of novelty is highly significant.

The authors make an excellent job at keeping up with current literature and their remarks and findings are clearly explained and of interest to broader audiences. However, the title as it reads seems a bit misleading and should include elements of the single-cell approach used. I almost reject reviewing it just by the title, but then I got very excited with the abstract.

The authors made clear statements of where the data and scripts will be deposited, and the methods section has clear statements of inclusivity and ethics.

I highly recommend (accept) this article for publication and just recommend tuning the title to make it more specific. 